\title{
TAMIZAJE DE ASPECTOS PSICO-ONCOLÓGICOS: VALIDACIÓN DE UNA LISTA DE CHEQUEO
}

\author{
SCREENING OF PSYCHO-ONCOLOGIC ISSUES: VALIDATION OF A CHECKLIST
}

\author{
Oscar Galindo Vázquez', Mayelli Muñóz Martínez², Sofía Campos Ugalde², Edith Rojas \\ Castillo², María Cristina Maya Vázquez ${ }^{3}$ y Salvador Alvarado Aguilar². \\ 'Facultad de Psicología, Universidad Nacional Autónoma de México UNAM. \\ ${ }^{2}$ Servicio de Psico-oncología, Instituto Nacional de Cancerología. \\ ${ }^{3}$ Facultad de Medicina, Universidad Nacional Autónoma de México UNAM / Instituto Nacional de Psiquiatría \\ "Ramón de la Fuente Muñiz"
}

Resumen

Objetivo: Desarrollar y validar una Lista de Chequeo de aspectos psico- oncológicos (LCPsico-onco) en pacientes con cáncer en tratamiento médico.

Método: Se entrevistó a 200 pacientes del Instituto Nacional de Cancerología de México (INCAN). La obtención de la muestra fue por disponibilidad en el periodo comprendido de Octubre de 2012 a Enero de 2013.

Resultados: El análisis factorial oblicuo presentó un modelo de tres factores y 2 indicadores con 16 reactivos. La consistencia interna de la escala global mostró un índice satisfactorio $(\alpha=0,811)$. Las alfas de Cronbach de cada subescala tuvieron un valor de 0,753 y 0,507 que explican el $43,81 \%$ de la varianza. La validez por correlación con medidas concurrentes mostró resultados significativos ( $r$ de Pearson de 0,51 a $0,68, p<0,05)$.

Conclusiones: La LC-Psico-onco presentó adecuadas características psicométricas. Al ser breve y fácil de aplicar tanto en la práctica clínica como en la investigación con población oncológica ofrece una guía en la planeación de evaluaciones exhaustivas y el diseño de un plan de tratamiento psicooncológico.
Abstract

Objective: Develop and validate a Checklist of psycho-oncologic issues (LC-Psycho-onco) for oncologic patients in treatment.

Method: We included 200 patients at the National Cancer Institute (INCAN). The sample collection was for availability during the period of October 2012 to January 2013.

Results: An oblique factor analysis presented a three-factor model with 16 indicators and two reagents. The internal consistency of the global scale showed a satisfactory index $(\alpha=0.811)$. The Cronbach's alphas for each subscale were worth 0.753 and 0.507 which explain $43.81 \%$ of the variance. The validity through the correlation with concurrent measures showed significant results (Pearson's $r=.51$ to $.68, p<0.05)$.

Conclusions: The LC-Psycho-onco showed adequate psychometric properties. Being a brief and easy instrument it is useful for both clinical practice and investigation. It provides a guide for planning psychological interventions.

Key words: Checklist, psychological assessment, validation, psycho-oncology, quality of life, cancer patients.

\section{Correspondencia:}

Men C. Oscar Galindo Vázquez

Servicio de Psico-oncología

Instituto Nacional de Cancerología

Av. San Fernando No. 22, Colonia. Sección XVI

México D.F., C.P. 14080

E-mail: psigalindo@yahoo.com.mx 
Palabras clave: Lista de chequeo, Evaluación psicológica, Validación, Psico-oncología, Calidad de vida, Pacientes con cáncer.

\section{INTRODUCCIÓN}

El cáncer es uno de los principales problemas de salud pública en México(1). Durante el proceso de enfermedad, afectaciones psicológicas clínicamente significativas constituyen una constante en la vida del paciente con cáncer ${ }^{(2)}$.

Diversas investigaciones muestran que del 15 al 58\% de la población oncológica presenta alguna afectación psicológica como sintomatología o trastorno; superior a lo reportado en la población genera| ${ }^{(3-7)}$.

Se han identificado los trastornos de ansiedad y depresión como los principales problemas psicológicos en pacientes con cáncer ${ }^{(8-10)}$. Además de trastornos del sueño, cambios en el apetito, fatiga, lentitud y/o agitación psicomotora, dificultad para concentrarse y dificultades en la toma de decisiones $^{(11)}$.

Dichas alteraciones pueden influir negativamente en la calidad de vida, duración de la estancia hospitalaria, autocuidado, adhesión al tratamiento médico, por lo que es fundamental su diagnóstico y su tratamiento ${ }^{(12-14)}$.

Una herramienta práctica y económica para la detección oportuna de las afectaciones psico-oncológicas, la constituyen instrumentos de tamizaje válidos y confiables ya que hacen posible la detección de probables casos clínicos.

El objetivo del presente estudio fue desarrollar y validar una lista de chequeo de aspectos psico-oncológicos (LC-Psicoonco) para pacientes con cáncer en tratamiento médico.

\section{EVALUACIÓN PSICO-ONCOLÓGICA}

El diagnóstico de cáncer puede ocasionar reacciones físicas y emocionales negativas ${ }^{(12)}$.
La Psico-oncología tiene un papel clave en la oncología. En las diferentes fases de la enfermedad, las áreas de trabajo más importantes son la evaluación, intervención, mejora y mantenimiento del paciente oncológico y su entorno(15).

Clark et al. (2011) ${ }^{(16)}$ mencionan que el sub-diagnóstico de las afectaciones psicosociales incide en un limitado apoyo psicosocial. Lo que hace necesario un tamizaje que permita identificar a aquellos pacientes oncológicos en riesgo de desarrollar alguna psicopatología ${ }^{(5)}$.

A pesar de las alteraciones psicológicas documentadas en este grupo de pacientes, la detección de síntomas no se produce de forma rutinaria ${ }^{(17,18)}$.

Entre las barreras para su detección se encuentran; el poco uso de instrumentos estandarizados y el desconocimiento en su aplicación, calificación e interpretación ${ }^{(16,19,20)}$.

La importancia de una adecuada detección y tratamiento de los problemas psicológicos es justificada por diversas razones: los pacientes oncológicos con mayores niveles de malestar psicológico requieren más servicios médicos, tienen mayor dificultad en la toma de decisiones, insatisfacción con la atención médica, menor adhesión al tratamiento, una baja adaptación a la enfermedad, calidad de vida deficitaria e incluso menor longevidad ${ }^{(16,21,22)}$.

Una adecuada detección de las afectaciones psicosociales permite realizar intervenciones psicológicas que han demostrado efectos benéficos ${ }^{(23-25)}$.

Ante la necesidad de diseñar y poner en marcha adecuados procedimientos de tamizaje, el presente trabajo tuvo por objetivo desarrollar y evaluar una Lista de Chequeo de aspectos psicosociales en pa- 
cientes oncológicos en tratamiento, que permita derivarles oportunamente a la atención psico-oncológica.

\section{MÉTODO}

\section{Sujetos}

Se incluyeron 200 pacientes del Instituto Nacional de Cancerología de México (INCAN). La obtención de la muestra fue por disponibilidad durante el periodo comprendido de Octubre de 2012 a Enero de 2013. Cada uno de los participantes aceptó participar en el presente estudio mediante la comprensión y firma del consentimiento informado de investigación.

Los participantes fueron seleccionados de manera intencional a partir de los siguientes criterios.

Criterios de inclusión: 1) Pacientes en tratamiento oncológico, 2) Cualquier tipo de diagnóstico y estadio oncológico y 3) Índice Karnofsky igual o mayor a 50.

Criterios de exclusión: 1) Pacientes que presentaran cuadros psiquiátricos severos y/o adicción a alguna sustancia psicoactiva, y 2) Con complicaciones vasculares mayores, deficiencias cognitivas así como problemas auditivos y visuales severos.

Se determinaron las variables sociodemográficas y clínicas de los pacientes con base en el expediente clínico electrónico IncanetG2.

\section{Medición concurrente}

\section{EORTC -QLQ-C30}

El instrumento de Calidad de vida de la Organización Europea para la investigación y tratamiento del cáncer, Aaronson et al. $(2003)^{26}$ es un instrumento evaluado y validado en estudios multinacionales y multiculturales. Validado en población mexicana por Oñate-Ocaña et al. (2009 (27), Está diseñado como un instrumento autoadministrable, que el paciente puede con- testar en aproximadamente 10 minutos. Evalúa cinco escalas funcionales: 1.física, 2.de desempeño, 3.cognitiva, 4.emocional y 5.social); tres escalas sintomáticas: 1.fatiga, 2.dolor y 3.náusea/vómito; una escala global de calidad de vida y otros síntomas que los pacientes oncológicos presentan frecuentemente.

\section{Procedimiento}

Lista de Chequeo - (LC-Psico-onco)

Fase I

Desarrollo de reactivos

Mediante revisión de la literatura se desarrolló una versión preliminar de 57 reactivos, correspondientes con las principales afectaciones psicosociales y síntomas físicos evaluados por la Escala de ansiedad y depresión Hospitalaria HADS(28), el Inventario de Calidad de vida y salud INCAVISA ${ }^{(29)}$ y el Termómetro de malestar emocional(30)

Fase II

Validación por jueces

Diez jueces expertos en el área de la psicología de la salud evaluaron a través de un formato de jueceo la validez de contenido, de facie, redacción correcta, identificación de palabras confusas, dificultades en la comprensión de las instrucciones y los ítems propuestos.

Resultando de éste proceso una lista de chequeo compuesta de tres partes: La primera, una cédula de identificación que recaba datos como: nombre, sexo, estado civil, paternidad, escolaridad, conocimiento del diagnóstico, tiempo transcurrido desde el diagnóstico, conocimiento del tratamiento recibido y objetivo del mismo. La segunda, con 26 reactivos que evalúan 8 áreas (1. estado de ánimo, 2. preocupaciones, 3. afrontamiento, 4. actitud ante el tratamiento, 5. apoyo social, 6. calidad de vida, 7. preocupaciones adicionales a la enfermedad y 8 . molestias físicas); deter- 
minando la severidad con que se presentan a través de las opciones de respuestas: Nunca, A veces y Siempre. Las categorías corresponden a síntomas que se incluyen en el diagnóstico de alguna afectación psicológica y/o dificultades psicosociales reportadas en la literatura. La tercera, incluye una lista de chequeo de 10 síntomas frecuentes en pacientes oncológicos.

Posteriormente se realizó una prueba piloto de la versión revisada a 15 pacientes en tratamiento médico, en diferentes etapas de la enfermedad. Aplicando un formato de registro de caso que incluyó preguntas sobre elementos confusos, ofensivos y comprensión de los reactivos.

Se realizaron modificaciones menores en cuanto a redacción de los ítems.

\section{Fase III}

Aplicación de la LC-Psico-onco

Residentes de Psico-oncología realizaron la entrevista y aplicación de los instrumentos en los Servicios de Oncología Médica, Cirugía, Hematología, Radioterapia y Psico-oncología; en consulta externa y hospitalización.

\section{Análisis Estadístico}

Se efectuó estadística descriptiva para caracterizar a la muestra con base en los datos sociodemográficos y clínicos.

Para el análisis factorial, se realizó: a) análisis descriptivos de coeficientes, estadísticos de $\mathrm{KMO}(0,787)$ y prueba de esfericidad de Bartlett $(\mathrm{P}<0.05)$, b) método de extracción de máxima verosimilitud y mínimos cuadrados ponderados y c) método de rotación oblicua Promax $(K=4)$ con un punto de corte de los coeficientes de los ítems de 30 .

Se llevó a cabo correlación productomomento de Pearson para la asociación con los diferentes componentes de la LCPsico-onco y EORTC QLQ-30, con un nivel de confianza de $95 \%$.

\section{RESULTADOS}

El $41 \%$ del grupo de participantes tenía seis meses o menos de tratamiento, 37,5\% más de un año y 21,5\% de siete meses a un año. Los síntomas físicos más frecuentes fueron cansancio y dolor. El 37,5 \% de los participantes presentó un Índice Karnofsky de 90, 27,5\% de 100, 23,5\% de 80 y 11,5 entre 50 y 70 , ver tabla I.

\section{Estructura factorial}

El análisis factorial oblicuo indicó un modelo de tres factores (Estado cognitivo, Preocupaciones y Afrontamiento al tratamiento) y dos indicadores (Ansiedad/Depresión y Relación familiar) que explican el 43,81\% de la varianza, ver tabla 2 .

Los reactivos sobre apoyo social y afrontamiento, fueron eliminados debido a que no permanecieron en ninguno de los factores e indicadores principales.

Sin embargo por su relevancia clínica, se reformularon y se incluyeron en la cédula de identificación.

\section{Consistencia interna}

El alfa de Cronbach total obtenida fue de 0.811, por subescalas: 1. Estado de ánimo $(5$ reactivos $)=0,753,2$. Preocupaciones (4 reactivos) $=0,760 ; 3$. Afrontamiento al tratamiento (3 reactivos) $=0,593,4$. Ansiedad/Depresión (2 reactivos) $=0,593 \mathrm{y}$ 5. Relación familiar (2 reactivos) $=0,507$. Las estimaciones se consideran adecuadas.

\section{Validez externa}

La asociación entre las subescalas de la LC-Psico-onco y las medidas concurrentes del EORTC fueron positivas y estadísticamente significativas ( $r$ de Pearson de 0,15 a $0,51, p<0,05)$. El indicador 1 correlacionó con 5 subescalas y el 2 con $1(p<0,05)$ del EORTC QLQ30, ver tabla 3. 
Tabla 1. Descripción de la muestra

\begin{tabular}{|c|c|c|c|c|c|}
\hline & $f$ & $\%$ & & $f$ & $\%$ \\
\hline $\mathbf{N}$ & 200 & 100 & Diagnóstico & & \\
\hline \multirow[t]{2}{*}{ Edad (Rango) 17-87 } & & & Mama & 62 & 32,0 \\
\hline & & & Ginecológicos & 32 & 16,0 \\
\hline Sexo & & & Hematológicos & 28 & 14,0 \\
\hline Masculino & 72 & 36,0 & Urogenitales & 20 & 10,0 \\
\hline \multirow[t]{2}{*}{ Femenino } & 128 & 64,0 & Pulmón & 17 & 8,5 \\
\hline & & & Gástricos & 11 & 5,5 \\
\hline Escolaridad & & & Cabeza y cuello & 9 & 4,5 \\
\hline Ninguno & 23 & 11,5 & Neoplasias de la piel & 8 & 2,5 \\
\hline Primaria & 46 & 23,0 & Otros & 13 & 7,0 \\
\hline Secundaria & 40 & 20,0 & & & \\
\hline Bachillerato & 58 & 29,0 & Estadio Clínico & & \\
\hline Licenciatura & 27 & 13,5 & I & 25 & 12,5 \\
\hline Posgrado & 6 & 3,0 & II & 49 & 24,5 \\
\hline $\begin{array}{l}\text { Estado civil } \\
\text { Soltero(a) }\end{array}$ & 56 & 28,0 & $\begin{array}{l}\text { III } \\
\text { IV } \\
\text { Tratamiento }\end{array}$ & $\begin{array}{l}75 \\
51\end{array}$ & $\begin{array}{l}37,5 \\
25,5\end{array}$ \\
\hline Casado(a) & 107 & 53,5 & QT & 90 & 45,0 \\
\hline Divorciado(a) & 16 & 8,0 & Cirugía & 23 & 11,5 \\
\hline Viudo(a) & 6 & 3,0 & RT & 22 & 11,0 \\
\hline \multirow[t]{5}{*}{ Unión libre } & 15 & 7,5 & $\mathrm{QT}+\mathrm{RT}$ & 17 & 8,5 \\
\hline & & & Cirugía + RT & 8 & 6,3 \\
\hline & & & Cirugía + QT & 39 & 4,0 \\
\hline & & & Otros & 16 & 5,7 \\
\hline & & & Comprensión parcial del Tx & 15 & 8,0 \\
\hline
\end{tabular}

Nota: QT= Quimioterapia, RT=Radioterapia.

De acuerdo a los resultados, se propone una versión de la LC-Psico-onco con 3 factores (Funcionamiento cognitivo, Preocupaciones y Afrontamiento ante el tratamiento) y dos indicadores (Ansiedad-Depresión e Interacción familiar) con 16 ítems.
La versión final del LC-Psico-onco se complementa con una cédula de identificación de aspectos demográficos y clínicos, de un apartado de síntomas físicos, impresión diagnóstica, reevaluación psicooncológica y posible intervención. 
Tabla 2. Análisis factorial de elementos

\begin{tabular}{|c|c|c|c|}
\hline $\begin{array}{l}\text { Escala Global } \alpha=0,811 \\
\text { Varianza Explicada }=43,81 \%\end{array}$ & $\begin{array}{l}\text { Peso } \\
\text { factorial }\end{array}$ & $\begin{array}{l}\text { Varianza } \\
\text { Explicada }\end{array}$ & $\begin{array}{l}\text { Alfa de } \\
\text { Cronbach }\end{array}$ \\
\hline $\begin{array}{l}\text { Factor 1: Estado cognitivo } \\
\text { Le cuesta trabajo seguir las } \\
\text { indicaciones sobre su tratamiento }\end{array}$ & 0,730 & 16,323 & $\alpha=0,753$ \\
\hline $\begin{array}{l}\text { Ha tenido problemas con su memoria } \\
\text { y concentración }\end{array}$ & 0,669 & & \\
\hline $\begin{array}{l}\text { Siente que es una carga para los } \\
\text { demás }\end{array}$ & 0,598 & & \\
\hline $\begin{array}{l}\text { Ha sentido que las personas no } \\
\text { entienden lo que está viviendo }\end{array}$ & 0,585 & & \\
\hline $\begin{array}{l}\text { Ha perdido interés en realizar } \\
\text { actividades que normalmente le } \\
\text { generaban placer }\end{array}$ & 0,416 & & \\
\hline $\begin{array}{l}\text { Factor 2: Preocupaciones } \\
\text { Se siente preocupado(a) por los } \\
\text { resultados de sus estudios }\end{array}$ & 0,691 & 13,653 & $\alpha=0,760$ \\
\hline $\begin{array}{l}\text { Se siente preocupado(a) por su } \\
\text { tratamiento }\end{array}$ & 0,686 & & \\
\hline Le preocupa su futuro & 0,632 & & \\
\hline Le preocupa su situación económica & 0,623 & & \\
\hline Factor 3: Afrontamiento al tratamiento & & 6,068 & $\alpha=0,593$ \\
\hline $\begin{array}{l}\text { En algún momento ha pensado dejar } \\
\text { el tratamiento }\end{array}$ & 0,534 & & \\
\hline $\begin{array}{l}\text { Le resulta difícil sobrellevar las } \\
\text { molestias ocasionadas por el } \\
\text { tratamiento médico }\end{array}$ & 0,506 & & \\
\hline $\begin{array}{l}\text { Piensa que desde que está enfermo(a) } \\
\text { las cosas le salen mal }\end{array}$ & 0,454 & & \\
\hline Indicador 1: Ansiedad/Depresión & & 4,667 & $\alpha=0,593$ \\
\hline $\begin{array}{l}\text { Se siente triste o deprimido(a) la mayor } \\
\text { parte del tiempo }\end{array}$ & 0,951 & & \\
\hline $\begin{array}{l}\text { Se ha sentido tenso(a), nervioso(a) la } \\
\text { mayor parte del tiempo }\end{array}$ & 0,319 & & \\
\hline Indicador 2: Relación familiar & & 3,099 & $\alpha=0,507$ \\
\hline Le preocupa la relación con su pareja & 0,720 & & \\
\hline $\begin{array}{l}\text { Le preocupa el cuidado que les da a } \\
\text { sus hijos }\end{array}$ & 0,458 & & \\
\hline
\end{tabular}




\section{Tabla 3. Correlaciones entre LC-Psicoonco y EORTC-QLQ30}

\begin{tabular}{|c|c|c|c|c|c|}
\hline \multirow[b]{2}{*}{ EORTC QLC-C30 } & \multicolumn{5}{|c|}{ LISTA DE CHEQUEO } \\
\hline & $\begin{array}{l}\text { Factor } 1 \\
\text { Estado } \\
\text { cognitivo }\end{array}$ & $\begin{array}{c}\text { Factor } 2 \\
\text { Preocupaciones }\end{array}$ & $\begin{array}{c}\text { Factor } 3 \\
\text { Afrontamiento } \\
\text { al tratamiento }\end{array}$ & $\begin{array}{c}\text { Indicador } \\
4 \\
\text { Ansiedad/ } \\
\text { Depresión }\end{array}$ & $\begin{array}{c}\text { Indicador } 5 \\
\text { Relaciones } \\
\text { familiares }\end{array}$ \\
\hline $\begin{array}{l}\text { 1. Funcionamiento } \\
\text { físico }\end{array}$ & $0,328^{* *}$ & $0,278^{* *}$ & $0,368^{* *}$ & $0,216^{* *}$ & 0,042 \\
\hline $\begin{array}{l}\text { 2. Funcionamiento } \\
\text { de rol }\end{array}$ & $0,153^{*}$ & $0,176^{*}$ & $0,222^{* *}$ & 0,038 & 0,004 \\
\hline $\begin{array}{l}\text { 3. Funcionamiento } \\
\text { emocional }\end{array}$ & $0,405^{* *}$ & $0,470^{* *}$ & $0,398^{* *}$ & $0,607^{* *}$ & $0,151^{*}$ \\
\hline $\begin{array}{l}\text { 4. Funcionamiento } \\
\text { cognitivo } \\
\end{array}$ & $0,511^{* *}$ & $0,326^{* *}$ & $0,230^{* *}$ & $0,343^{* *}$ & 0,087 \\
\hline $\begin{array}{l}\text { 5. Funcionamiento } \\
\text { social }\end{array}$ & $0,290^{* *}$ & $0,347^{* *}$ & $0,329^{* *}$ & $0,281^{* *}$ & 0,090 \\
\hline $\begin{array}{l}\text { 6. Dificultades } \\
\text { financieras }\end{array}$ & $0,220^{* *}$ & $0,321^{* *}$ & $0,232^{* *}$ & $0,259^{* *}$ & $-0,069$ \\
\hline
\end{tabular}

**. Correlaciones Pearson con $p<0,01$.

* Correlaciones Pearson con $p<0,05$.

\section{DISCUSIÓN Y CONCLUSIONES}

La Lista de Chequeo LC-Psico-onco resulta una herramienta confiable y válida para la identificación de afectaciones psicológicas. Al ser breve y fácil de aplicar resulta útil en la práctica clínica y en la investigación con población oncológica.

Su uso ofrece una guía en la planeación de una evaluación exhaustiva y el diseño de un plan de tratamiento psicooncológico.

La evaluación de los factores emocionales asociados al proceso de cáncer y la oportuna derivación de los pacientes a los servicios de salud mental repercutirá en un mejor afrontamiento de la enfermedad y sus tratamientos.

Se observó que el $8 \%$ de los participantes comprende parcialmente el tratamiento que recibe por lo que la aplicación de la LC-Psico-onco puede coadyuvar en el manejo de información durante el proceso de atención.
Se sugiere que los pacientes sean evaluados a su ingreso y periódicamente durante el proceso de atención. Por ejemplo, al inicio y término de un tratamiento, en el periodo de seguimiento y en la derivación a una unidad de cuidados paliativos ${ }^{21}$.

\section{Limitaciones y sugerencias}

Se requerirá en futuros estudios el empleo de una muestra mayor, así como un diseño prospectivo que permita confirmar las propiedades psicométricas de la LCPsico-onco.

\section{REFERENCIAS BIBLIOGRÁFICAS}

1. Galindo O, Álvarez MA, Alvarado S. Aspectos psicológicos de las afectaciones sexuales en el paciente con cáncer testicular. Rev Mex Urol 2012;72:256-63.

2. Spiegel D. Cancer and depression. Br J Psychiatry 1996;168:109-16. 
3. Maté J, Hollenstein M, Gil F. Insomnio, ansiedad y depresión en el paciente oncológico. Psicooncología 2004;1:211-30.

4. Derogatis LR, Morrow GR, Fetting J. The prevalence of psychiatric disorders among cancer patients. JAMA 1983;249:751-7. Doi:10.1001/ jama.1983.03330300035030.

5. Jadoon NA, Munir W, Shahzad MA, Choudhry ZS. Assessment of depression and anxiety in adult cancer outpatients: A crosssectional study. BMC Cancer 2010;10:594. Doi:10.1186/1471-2407-10-594

6. Hinz A, Krauss HA, Hauss JP, Höckel M, Kortmann RD, Stolzenburg JU, et al. Anxiety and depression in cancer patients compared with the general population. Eur J Cancer Care 2010;19:522-9. Doi: 10.1111/j.1365-2354.2009.01088.x

7. Massie M.J. Prevalence of depression in patients with cancer. J Natl Cancer Inst Monogr 2004;32;57-71. Doi: 10.1093/jncimonographs/lgh014

8. Pirl WF. Evidence report on the occurrence, assessment, and treatment of depression in cancer patients. J Natl Cancer Inst Monogr 2004;32:32-9. Doi: 10.1093/jncimonographs/lgh026

9. Zabora J, Brintzenhofe Szoc K, Curbow B, Hooker C, Piantadosi S. The prevalence of psychological distress by cancer site. Psychooncology 2001,10:19-28. Doi: 10. 1002/1099-1611(200101/02)10:1<19::AI D-PON501>3.0.CO;2-6

10. Montazeri A, Milroy R, Hole D, McEwean J, Gills CR. Anxiety and depression in patients with lung cancer before and after diagnosis: findings from a population in Glasgow, Scotland. J Epidemiol Community Health 1998; 52:203-4.

11. Mehnert A, Koch U. Psychological comorbidity and health-related quality of life and its association with awareness, utilization, and need for psychosocial support in a cancer register-based sample of long-term breast cancer survivors. J Psychosom Res 2008;64:383-91
12. Holland JC. Psycho-oncology. New York: Oxford University Press; 1998.

13. Vahdaninia M, Omidvari S, Montazeri A. What do predict anxiety and depression in breast cancer patients? A follow-up study. Soc Psychiatr Epidemiol 2010;45:355-61. Doi: 10.1007/s00127-009-0068-7

14. Bottomley A. Depression in cancer patients: A literature review. Eur J Cancer 1998;7:181-91. Doi:10.1046/j.13652354.1998.00100.x

15. Arraras JI, Martínez M, Manterota A, Laínez N. La evaluación de la calidad de vida del paciente oncológico. el grupo de calidad de vida de la EORTC. Psicooncología 2004;1:87-98.

16. Clark PG, Rochon E, Brethwaite D, Edmiston KK. Screening for psychological and physical distress in a cancer inpatient treatment setting: A pilot study. Psychooncology 2011;20:664-8. Doi:10.1002/pon.1908

17. Hack TF, Degner LF, Parker PA. The communication goals and needs of cancer patients: A review. Psychooncology 2005;14:831-45. Doi:10.1002/pon.949

18. Institute of Medicine (IOM). Cancer care for the whole patient: Meeting psychosocial health needs. The National Academies Press: Washington, DC, 2008.

19. Muriel AC, Hwang VS, Kornblith A Greer J, Greenberg DB, Temel J, et al. Management of psychosocial distress by oncologists. Psychiatr Serv 2009;60:1132-4. Doi: 10.1176/appi.ps.60.8.1132

20. Jacobsen PB, Ransom S. Implementation of NCCN distress management guidelines by member institutions. J Natl Compr Canc Netw 2007;5:99-103. Doi:10.1200/ JCO.2007.13.1367

21. Carlson LE, Bultz BD. Cancer distress screening: Needs, models, and methods. J Psychosom Res 2003;55:403-9. Doi:10.1016/S0022-3999(03)00514-2

22. Nordin K, Berglund G, Glimelius B, Sjödén PO. Predicting anxiety and depression among cancer patients: A clinical 
model. Eur J Cancer 2001;37:376-84. Doi:10.1016/j.bbr.2011.03.031.

23. Sellick SM, Edwardson AD. Screening new cancer patients for psychological distress using the hospital anxiety and depression scale. Psychooncology 2007;16:534-42. Doi:10.1002/pon.1085

24. Tatrow K, Montgomery GH. Cognitive behavioral therapy techniques for distress and pain in breast cancer patients: A meta-Analysis. J Behav Med 2010;29:17-27. Doi:10.1007/s10865-005-9036-1

25. Penedo FJ, Traeger L, Dahn J, Molton I, Gonzalez JS, Schneiderman N. et al. Cognitive behavioral stress management intervention improves quality of life in Spanish monolingual Hispanic men treated for localized prostate cancer: Results of a randomized controlled trial. Inter J Behav Med 2007;14:164-72.

26. Aaronson, N. K., Ahmedzai, S., Bergman, B., Bullinger, M., Cull, A., Duez, N. J. et al. The European Organization for Research and Treatment of Cancer QLQC30: A quality-of-life instrument for use in international clinical trials in oncology. J Natl Cancer Inst 1993;85:365-76. Doi: 10.1093/jnci/85.5.365

27. Oñate-Ocaña LF, Alcántara-Pilar A, VilarCompte D, Garcia-Hubard G, Rojas-Castillo E, Alvarado-Aguilar S, et al. (2009). Validation of the Mexican Spanish version of the EORTC C30 and STO22 questionnaires for the evaluation of health-related quality of life in patients with gastric cancer. Annals Surg Oncol 2009;16:88-95. Doi:10.1245/s10434-008-0175-9

28. Zigmond AS, Snaith RP. The Hospital Anxiety and Depression Scale. Acta psychiatr Scandinavic 1983;67:361-70. Doi:10.1111/j.1600-0447.1983.tb09716.x

29. Riveros A, Sánchez-Sosa JJ, Groves M. Inventario de calidad de vida y salud. InCaVisa: México Universidad Nacional Autónoma de México, 2004.

30. Almanza MJJ, Rosario JI, Pérez S. Traducción, adaptación y validación del Termómetro de Distrés en una muestra de pacientes mexicanos con cáncer. Rev Sanid Milit Mex 2008;62:209-17. 
